\title{
Joint Coordination When Running in Minimalist, Neutral, and Ultra-Cushioning Shoes
}

\section{Authors: Brianne Borgia, Julia Freedman Silvernail, and James Becker}

This is an Accepted Manuscript of an article published by Taylor \& Francis in 'Journal of Sports Sciences' on 2020-03-13, available online: https:// www.tandfonline.com/10.1080/02640414.2020.1736245.

Borgia, Brianne, Julia Freedman Silvernail, and James Becker. "Joint Coordination When Running in Minimalist, Neutral, and Ultra-Cushioning Shoes." Journal of Sports Sciences 38, no. 8 (March 13, 2020): 855-862. doi:10.1080/02640414.2020.1736245. 


\title{
Joint Coordination When Running in Minimalist, Neutral, and Ultra-Cushioning Shoes
}

\author{
Brianne Borgia ${ }^{1}$, Julia Freedman Silvernail ${ }^{1}$, James Becker ${ }^{2, *}$ \\ ${ }^{1}$ Department of Kinesiology and Nutrition Sciences, University of Nevada, Las Vegas, Las \\ Vegas, NV 89154, USA \\ ${ }^{2}$ Department of Health and Human Development, Montana State University, Bozeman, MT, \\ 59717, USA
}

\author{
Corresponding Author \\ Dr. James Becker, PhD \\ 121 Hoseaus Fitness Complex \\ Montana State University \\ Bozeman, MT 59717 \\ Email address: james.becker4@montana.edu
}


Joint Coordination When Running in Minimalist, Neutral, and Ultra-Cushioning Shoes 


\begin{abstract}
Ultra-cushioning (ULTRA) shoes are relatively new to the running shoe market. While several studies have evaluated joint kinematics and kinetics when running in ULTRA shoes, to date it remains unknown how such shoes influence joint coordination. Therefore the purpose of this study was to evaluate lower extremity coordination and coordination variability when running in minimalist (MIN), traditional (NEUT) and ULTRA shoes. Fifteen recreational runners ran for ten minutes in each shoe type. Lower extremity coordination patterns and coordination variability were assessed for rearfoot-tibia, rearfoot-knee, and tibia-knee couplings using a modified vector coding method during early, mid, and late stance periods. During late stance the ULTRA shoes results in more antiphase coordination than the MIN $(p=.036)$ or NEUT ( $p$ $=.047)$ shoes and less in-phase coordination than the MIN $(p=.048)$ or NEUT $(p=.013)$ shoes. During late stance there was also more proximal phase rearfoot-knee coordination in the ULTRA shoes than in either the MIN $(p=.039)$ or the NEUT $(p=.005)$ shoes and less in-phase coordination in the ULTRA shoes than in the NEUT shoes $(p=.006)$. There were no differences in coordination variability between shoes during any phase. The differences in coordination may have implications for tissue loading and injury development when running in ULTRA shoes.
\end{abstract}

Keywords: running, coordination, vector coding, footwear, minimalist, ultra-cushioning Word Count: 3,555 words. 


\section{Introduction}

Running is a popular form of exercise, with an estimated 19 million individuals running 3 or more days per week in the United States. However, within a given year about half of all runners will sustain a running related injury. ${ }^{1}$ Risk factors for sustaining an overuse injury include extrinsic factors such as duration and frequency of running ${ }^{2}$, running surfaces ${ }^{3}$ and shoes used $^{4}$ as well as intrinsic factors such as malalignments of the lower extremity or abnormal biomechanics..$^{5}$ Despite the substantial volume of research investigating running injuries, a detailed understanding of the cause of these injuries is still unknown.

One area which has received considerable attention in regards to the development of running injuries is how movement is coordinated across multiple joints in the lower extremity. Within this focus area several methodologies have been used. Initially, studies examined the relative timing of discrete events during stance phase. ${ }^{6}$ For example, numerous studies have compared the relative timing of peak rearfoot eversion and peak knee flexion with the assumption that prolonging subtalar eversion beyond peak knee flexion creates opposing torques acting on the proximal and distal ends of the tibia, thereby increasing stress on the knee. ${ }^{7-10}$ More recent studies have shifted away from looking at relative timing of discrete events and instead examined coordination patterns or coordination variability across the entire stance phase using dynamical systems techniques such as continuous relative phase (CRP) or vector coding. ${ }^{11-13}$ Compared to the discrete events approach, these methods allow for continuous evaluation of coordination patterns and coordination variability across the entire gait cycle, not just at specific points. ${ }^{11}$ Additionally, analysis of the coordination between adjacent segments surrounding a joint may reveal information regarding the way in which stress is applied to the joint or adjacent tissues. ${ }^{13}$ For example, low coordination variability has been reported in runners with numerous 
injuries including patellofemoral pain syndrome, lower back pain, and tibial stress fractures. ${ }^{11,14-}$ 16

Footwear is one mechanism which may influence lower extremity coordination, and by extension, injury risk. Previous research has examined the effect of footwear on lower extremity coordination using both discrete event timing and continuous coordination analyses, with mixed results. Hamill et al. ${ }^{7}$ compared relative timing of peak knee flexion and rearfoot eversion in shoes of three different hardness and found there were no differences between a hard and medium firmness insole, but that a softer insole disrupted the relative timing. In contrast, Stergiou and Bates $^{8}$ reported no differences in relative timing of peak knee flexion and peak rearfoot eversion when running in standard shoes on extra hard, hard, medium, and soft surfaces. Using CRP, Kurz and Stergiou ${ }^{17}$ reported no differences in sagittal or frontal plane foot-shank coordination patterns when running in hard or soft shoes. However, when comparing the shod conditions to running barefoot they found sagittal plane foot-shank coordination was more out of phase while frontal plane foot-shank coordination was more in-phase. Silvernail et al. ${ }^{4}$ used vector coding to examine forefoot-rearfoot coordination when running in motion control shoes compared to racing flats. While both shoes demonstrated relative forefoot eversion, during midstance movement in a motion control shoe showed more antiphase coordination while in a racing flat there was more in-phase coordination.

To date, studies on the influence of footwear on coordination have focused on comparisons between barefoot, minimalist, or traditional running shoes with varying midsole durometers. Relatively new to the market are so called "ultra-cushioning" shoes. These shoes feature an especially thick midsole and are purported to provide additional cushioning and shock attenuation compared to traditional running shoes. While these shoes are rapidly growing in 
popularity, relatively little is known regarding how they affect lower extremity mechanics during running. While a few studies have examined kinematic and kinetic differences between minimalist, traditional, and ultra-cushioning shoes ${ }^{18-22}$, to date no studies have reported how these shoes influence lower extremity coordination patterns. Therefore, the purpose of this study was to examine differences in inter-segment coordination and coordination variability when running in minimal, neutral, and ultra-cushioning shoes. Previous studies have reported that runners display differences in relative timing of joint motions and coordination variability when running in footwear with different midsole thickness. ${ }^{4,7}$ Due to the thicker, more cushioned midsole we hypothesized that there would be more antiphase coordination and greater coordination variability in the ultra-cushioning shoes condition compared to the neutral or minimalist shoe conditions.

\section{Methods}

\subsection{Participants}

Fifteen healthy, recreational runners ( 7 males with average age: $24.8 \pm 3.7$ years, mass: $67.6 \pm 8.5 \mathrm{~kg}$, height: $1.65 \pm 0.09 \mathrm{~m}$; and 8 females with average age: $24.8 \pm 3.6$ years, mass: $57.7 \pm 3.0 \mathrm{~kg}$ and height: $1.61 \pm 0.04 \mathrm{~m}$ ) participated in this study. All participants trained a minimum of three times/week, running at least 20 miles/week, and were injury free 6-months prior to and at the time of testing. Based on their completion of physical activity readiness and health history questionnaires, all participants were considered low-risk for participating in physical activity according to the American College of Sports Medicine. The protocols for the

study were approved by the University Institutional Review Board and all participants provided written informed consent prior to participation. 


\subsection{Shoe conditions}

The shoes used in this study were a minimal cushioning, light weight racing flat (MIN: New Balance 1400v3), a traditional, neutral cushioning shoe (NEUT: Nike Air Zoom Pegasus 32), and an ultra-cushioning shoe (ULTRA: Hoka OneOne Bondi 4). Participants were provided a pair of each type of shoe in their self-reported size. All shoes were new and had never been run in prior to the start of this study.

\subsection{Protocol}

Data collection took place in a single testing session during which each participant ran in each of the three shoe conditions. The order of the shoes was randomized. A total of 40 reflective markers were placed on the lower extremity. Anatomical markers defining rigid segments were placed bilaterally on the medial and lateral malleoli, medial and lateral femoral epicondyles, anterior superior iliac spine, and posterior superior iliac spine. Additional tracking markers were placed on the iliac crests, base of the 5th metatarsal, and head of the 2nd metatarsal, two markers along the vertical bisection of the heel counter, one on the lateral side of the heel counter. Lastly, rigid clusters of four non collinear markers were placed on the lateral sides of the thigh and shank segments.

Three static standing calibrations were collected prior to the first running trial, one for each footwear condition, after which the medial malleoli and femoral epicondyle markers were removed. Running kinematics were recorded using a 12-camera motion capture system (Qualisys, Inc., Gothenburg, Sweden) sampling at $200 \mathrm{~Hz}$ while participant ran on an instrumented treadmill (Bertec Corp., Columbus $\mathrm{OH}$ ) at $3.0 \mathrm{~m} / \mathrm{s}$. This speed was selected as it closely matched the speed participants reported using for their regular easy training runs. Ground reaction forces from the treadmill were sampled at 1,000 Hz. Participants ran for 10 minutes 
with each shoe condition at a speed of $3.0 \mathrm{~m} / \mathrm{s}$. Each of the participants regularly wore traditional running footwear and were not familiar with maximalist or minimalist footwear. Therefore, the first 9 minutes were used for familiarization to the footwear and data were collected during the last minute. Marker trajectories were recorded for thirty seconds, from which ten consecutive steps were selected for analysis. The speed of $3.0 \mathrm{~m} / \mathrm{s}$ was chosen to ensure completion of each 10-minute session without the participant experiencing fatigue. Additionally, between each footwear condition participants were allowed a 5-minute rest period during which they changed shoes.

\subsection{Data Analysis}

Raw marker trajectories and ground reaction forces were exported to Visual 3D (CMotion, Inc., Rockville MD) where they were filtered using 4th order, zero lag, low-pass Butterworth filters with cutoff frequencies of $6 \mathrm{~Hz}$ and $50 \mathrm{~Hz}$, respectively. For ten consecutive trials knee joint angle and segment angles of the tibia and rearfoot were calculated. Knee joint angles were calculated using a rotation matrix describing the orientation of the tibia segment relative to the femur segment using an $\mathrm{X}$ (flexion/extension), $\mathrm{Y}$ (abduction/adduction), $\mathrm{Z}$ (internal/external rotation) Cardan rotation sequence. Segment angles for the tibia and rearfoot were calculated using a rotation matrix describing the orientation of the segment relative to the fixed laboratory coordinate system using the same Cardan rotation sequence. Joint and segment angles were normalized to $100 \%$ of stance phase, with foot contact and toe off being identified based on when the vertical ground reaction force rose above, then fell below, a $50 \mathrm{~N}$ threshold, respectively.

Normalized joint and segment angles were exported to a custom LabVIEW program (National Instruments, Austin TX). For each of the joint and segment angles, the position at 
initial contact, peak position, and range of motion were calculated. A modified vector coding (Chang, Van Emmerik, \& Hamill, 2008) approach was used to determine the segment couplings of: rearfoot-tibia, tibia-knee, and rearfoot-knee. Angle-angle plots were constructed for each coordination pattern, with the proximal segment on the $\mathrm{x}$-axis and the distal segment or joint on the y-axis. Coupling angles were calculated based on the orientation of a vector connecting two consecutive points on the angle-angle plots relative to the right horizontal. This procedure was continued for all successive points to produce coupling angles at all 101 data points of the stance phase. Using $45^{\circ}$ bins, coupling angles at each point were categorized into one of four categories: proximal phase, in-phase, distal phase, or anti-phase (Table 1). ${ }^{23}$ The frequency (number of data points) at which each coordination pattern occurred was determined for three different time periods, early stance (ES: 0-33\%), midstance (MS: 34\% - 66\%), and late stance (LS: $67 \%-100 \%)$.

\section{** Insert Table 1 about here **}

For each participant and shoe condition, mean coupling angles during the ES, MS, and LS time periods were calculated using circular statistics. ${ }^{24}$ Variability in coordination patterns was determined by calculating the coupling angle variability. This entailed calculating the standard deviation in coupling angles at each point across stance phase, also using circular statistics. Mean coupling angle variability during the ES, MS, and LS periods was calculated as the average standard deviation of the coupling angles in the respective time period.

\subsection{Statistical Analysis}

Differences between shoe conditions in segment and joint positions and range of motion were evaluated using one way repeated measures analyses of variance (ANOVA). Within each portion of stance (ES, MS, and LS) differences in the frequencies with which a given 
coordination pattern occurred were evaluated using $3 \times 4$ (shoe $\mathrm{x}$ coordination pattern) repeated measures ANOVAS. Also within each portion of stance, differences in coupling angle variability were evaluated using one way repeated measures ANOVAs. When significant omnibus effects were observed, post-hoc comparisons were performed using Bonferroni corrections. All statistical analyses were done using Statistical Package for the Social Sciences (IBM Corp, Armonk NY), version 22, with level of significance set to $\mathrm{p}<.05$.

\section{Results}

\subsection{Kinematics}

There were no statistically significant differences in position at initial contact, peak position during stance, or range of motion for rearfoot segment, tibia segment, or knee joint (Table 2). The general pattern of sagittal plane motion at the knee, frontal plane motion at the rearfoot, and transverse plane motion of the tibia was similar across all shoe conditions (Figure 1).

$$
\text { ** Insert Figure } 1 \text { about here ** }
$$

\subsection{Rearfoot-Tibia Coordination}

Rearfoot-tibia coupling angles across stance are shown in Figure 2. During ES there was a significant shoe by coordination pattern interaction $\left(\mathrm{F}_{6,84}=3.508, p=.004, \eta^{2}=0.200\right)$ with proximal phase coordination occurring more often in the MIN shoes than the NEUT shoes ( $p$ $=.035)$ and in phase coordination occurring more often in the ULTRA shoes than in the NEUT shoes $(p=.019$, Figure 3$)$. There were no statistically significant differences in coordination pattern frequencies during MS (Figure 3). During LS there was also a significant shoe by coordination pattern interaction $\left(\mathrm{F}_{6,84}=3.001, p=.010, \eta^{2}=0.177\right)$. Antiphase coordination 
occurred more often in the ULTRA shoes than in either the MIN $(p=.036)$ or NEUT $(p=.047)$ shoes while in-phase coordination occurred less often in the ULTRA shoes than in either the MIN $(p=.048)$ or NEUT $(p=.013)$ shoes (Figure 3$)$. Coupling angle variability was not different between shoes during $\mathrm{ES}\left(\mathrm{F}_{2,28}=3.051, p=.063, \eta^{2}=0.179\right), \mathrm{MS}\left(\mathrm{F}_{2,28}=3.463, p\right.$ $\left.=.634, \eta^{2}=0.032\right)$, or LS $\left(F_{2,28}=3.1 .736, p=.195, \eta^{2}=0.110\right.$, Figure 4).

\subsection{Rearfoot-Knee Coordination}

Rearfoot-knee coupling angles across stance are shown in Figure 2. There were no statistically significant differences between shoes for coordination pattern frequencies during ES or MS (Figure 3). However, there was a significant shoe by coordination pattern interaction $\left(\mathrm{F}_{6,84}\right.$ $=5.610, p<.001, \eta^{2}=0.286$ ) during LS. Proximal phase coordination occurred more often in the ULTRA shoes than in either the MIN $(p=.039)$ or the NEUT $(p=.005)$ shoes and in-phase coordination occurred less often in the ULTRA shoes than in the NEUT shoes $(p=.006$, Figure 5). Coupling angle variability was not different between shoes during $\mathrm{ES}\left(\mathrm{F}_{2,28}=1.363, p\right.$ $\left.=.272, \eta^{2}=0.089\right), \operatorname{MS}\left(\mathrm{F}_{2,28}=1.028, p=.371, \eta^{2}=0.68\right)$, or $\operatorname{LS}\left(\mathrm{F}_{2,28}=0.678, p=.516, \eta^{2}=\right.$ 0.046, Figure 4).

\subsection{Tibia-Knee Coordination}

Tibia-knee coupling angles across stance are shown in Figure 2. There were no statistically significant differences between shoes for coordination pattern frequencies during ES

(Figure 3). However, there was a significant shoe by coordination pattern interaction during MS $\left(\mathrm{F}_{6,84}=3.552, p<.003, \eta^{2}=0.202\right)$. In-phase coordination occurred more often in the ULTRA shoes than in either the MIN $(p=.043)$ or NEUT $(p=.048)$ shoes (Figure 3$)$. There were no statistically significant differences between shoes for coordination patterns during LS (Figure 3). Coupling angle variability was not different between shoes during $\mathrm{ES}\left(\mathrm{F}_{2,28}=0.094, p=.910, \eta^{2}\right.$ 
$=0.007), \mathrm{MS}\left(\mathrm{F}_{2,28}=0.363, p=.699, \eta^{2}=0.025\right)$, or LS $\left(\mathrm{F}_{2,28}=0.091, p=.913, \eta^{2}=0.006\right.$,

Figure 4).

** Insert Figures 2, 3, and 4 about here **

\section{Discussion}

The purpose of this study was to investigate lower extremity coordination patterns and coordination variability in healthy runners while running on a treadmill in three different footwear conditions: minimalist, neutral, and ultra-cushioning shoes. To the author's knowledge, this is the first study to evaluate how ultra-cushioning shoes influence lower extremity coordination. We hypothesized there would be more antiphase coordination and greater coordination variability in the ULTRA shoes compared to the NEUT shoes. The data for the rearfoot-tibia coordination partially supports these hypotheses as during late stance anti-phase coordination occurred more often while in-phase coordination occurred less often in the ULTRA shoes than either the MIN or NEUT shoes. However, these same differences were not observed for rearfoot-knee or tibia-knee coordination patterns, and no differences in coordination variability between shoes were observed for any of the three coordination patterns.

During late stance the rearfoot-tibia demonstrated more antiphase and less in-phase coordination in the ULTRA shoes than in either the MIN or NEUT shoes. Translated into joint movements this means that there was more time when the tibia was internally rotating while the rearfoot was inverting. Also during late stance the rearfoot-knee demonstrated more proximal phase coordination in the ULTRA shoes than in either the MIN or NEUT shoes. The mean joint and segment kinematics (Figure 3) show that from approximately $90 \%$ of stance until toe off the knee flexed slightly. Thus, the mean rearfoot-knee coupling angles late in stance indicate this 
time period was dominated by knee flexion. This would also explain the tibia internal rotation, as motions of the knee and tibia are closely linked due to the anatomy of the tibiofemoral joint. ${ }^{25}$ Combined the rearfoot-tibia and rearfoot-knee coordination patterns suggest asynchrony between motions of the knee and subtalar joint during late stance when running in the ULTRA shoes compared to MIN and NEUT shoes. Previous research has suggested that this type of asynchrony may increase torsional stress on the knee and possibly play a role in the development of knee injuries. ${ }^{9}$ In this view ultra-cushioning shoes could therefore possibly increase risk of developing knee injuries. However, this requires further investigation as, to date, there are no prospective studies on injury incidence in runners who use ultra-cushioning shoes.

With the exception of the rearfoot-tibia and rearfoot-knee couplings during late stance, we observed few other statistically significant differences in coordination patterns between footwear conditions. This is consistent with previous studies using vector coding to assess the effects of footwear on lower extremity coordination. For example, while Silvernail et al. ${ }^{4}$ observed differences in forefoot-rearfoot coordination when running in motion control shoes compared to lightweight racing flats, they did not find any differences in coordination patterns for more proximal couplings including thigh-shank or shank-foot. This lack of differences can partially be explained by the preferred movement path paradigm. ${ }^{26}$ This paradigm proposes the body modulates muscle activity in order to maintain the same overall pattern of skeletal movement for a given task. In support of this hypothesis, it has been shown that the overall patterns of joint kinematics are similar when comparing running in racing flats, minimalist shoes, and traditional running shoes. ${ }^{27}$ This was also observed in the current study, where the overall patterns of knee flexion/extension, rearfoot eversion/inversion, and tibia internal/external rotation were similar across all three shoe types, with only small and non-statistically significant 
differences in peak angles. Given the calculations involved in the vector coding approach used to define coordination patterns, if the overall patterns of joint motion are similar between shoes then the coupling angles and coordination patterns should be similar as well. However, the preferred movement path paradigm also suggests that muscles might have to work more to keep the joint moving in the preferred movement path. ${ }^{26}$ If this is true then muscle activity might increase in order for the runner to maintain the same coordination pattern. While muscle activity was not evaluated in the current study, this would appear to be an area for future work, especially since to date, there is minimal literature examining the effects of changing muscle activity on coordination patterns.

In addition to the coordination patterns themselves, previous authors have highlighted the importance of variability in coordination patterns as this metric provides insight into the overall control of movement as well as potential injury mechanisms. ${ }^{11,14}$ While the were no statistically significant differences in coordination variability, there was a trend with a moderate effect size towards greater rearfoot-tibia coordination variability during early stance in the ULTRA shoes than in the MIN or NEUT shoes. Boyer et al. ${ }^{28}$ highlighted the importance of variability in tibia rotation-rearfoot eversion coordination both for impact force attenuation and knee injury risk. Runners with higher coordination variability during early stance would have more flexibility in how they attenuate impact forces, and thus, the trend towards higher rearfoot-tibia coordination variability in our participants might reflect a wider range of movement strategies for absorbing the impact with the ground when running in the ULTRA shoes. However, further investigation is required to confirm this hypothesis.

There are a few limitations to this study which must be considered when interpreting the results. First, while the three shoes used all had different properties, our minimalist shoes might 
be more accurately characterized as "partial minimalist" or "structured minimalist". Previous research has suggested there are little to no differences between partial minimalist shoes and traditional running shoes for joint kinematics..$^{29,30}$ This would also partially explain why there were only minimal differences in coordination patterns between shoes. Second, the acclimatization period participants received for each shoe was only the nine minutes of running prior to data being collected. As such, our results represent relatively acute influences on coordination. It is unknown whether runners would change their movement patterns, and by extension their coordination patterns, if a longer adaptation period was used. Similarly, none of our participants had previous experience running in either minimalist or ultra-cushioning shoes. Thus, we cannot be certain that habitual users of these shoes would not have adopted different coordination patterns over time. The shoes used in the current study were also new when the study began. Over time with mileage, the mechanical properties of shoes change, which can influence both kinematics and ground reaction forces during running. ${ }^{31,32}$ Thus, we cannot be certain that similar differences in coordination would be observed when running in used shoes. Finally, all the participants in this study were relatively highly experienced runners, logging more than twenty miles each week. Previous studies have reported that both endpoint variability and coordination variability differ between runners with different levels of experience. ${ }^{33-35}$ Whether similar results would be seen in more novice runners who have less coordination variability in their movement patterns, or whether experience level provides insight into the impact of different footwear on a runners movement, requires further investigation.

In summary, this study examined differences in lower extremity coordination patterns and coordination variability when running in minimalist, neutral, and ultra-cushioning shoes. We found that ultra-cushioning shoes have minimal influence on coordination patterns, with the 
major differences being in the rearfoot-tibia and rearfoot-knee coordination patterns during late stance. Similarly, these three types of shoes appear to have minimal effects on coordination variability. Combined, these results suggest that overall movement strategies are similar between these three types of shoes.

\section{Author Contributions}

BB conceived of and designed the study, performed data collection, processing and analysis, drafted the manuscript, and reviewed the final manuscript. JFS contributed to data processing and analysis as well as revisions and review of the final manuscript. JB contributed to design of the study, data collection, processing, and analysis, drafted the manuscript, and reviewed the final manuscript.

\section{Competing Interests}

None of the authors declare competing financial interests.

\section{Funding Sources}

This research did not receive any specific grant from funding agencies in the public, commercial, or not-for-profit sectors. 


\section{References}

1. Taunton JE, Ryan MB, Clement DB, et al. A retrospective case-control analysis of 2002 running injuries. Br J Sports Med. 2002;36(2):95-102.

2. Messier SP, Davis SE, Curl WW, Lowert RB, Pack RJ. Etiological factors associated with patellofemoral pain in runners. Med Sci Sports Exerc. 1991;23:1008-1015.

3. O'Connor KM, Hamill J. Does Running on a cambered road predispose a runner to injury? J Appl Biomech. 2002;(18):3-14.

4. Silvernail JF, Rohr E, Brueggemann P, Hamill J. Footwear and changes in lower extremity coordination during running. Footwear Sci. 2013;5(Sup1):S77-S78.

5. Rolf C. Overuse Injuries of the lower extremity in runners. Scand J Med Sci Sport. 1995;5(4):181-190.

6. DeLeo AT, Dierks TA, Ferber R, Davis IS. Lower extremity joint coupling during running: A current update. Clin Biomech. 2004;19(10):983-991.

doi:10.1016/j.clinbiomech.2004.07.005

7. Hamill J, Bates B, Holt KG. Timing of Lower Extremity Joint Actions During Treadmill Running. Med Sci Sport Exerc. 1992;24(7):807-813.

8. Stergiou N, Bates B. The Relationship Between Subtalar and Knee Joint Function as a Possible Mechanism for Running Injuries. Gait Posture. 1997;6:177-185.

9. Stergiou N, Bates BTT, James SL. Asynchrony Between Subtalar and Knee Joint Function During Running. Med Sci Sport Exerc. 1999;31(11):1645-1655.

10. Williams DS, McClay IS, Hamill J, Buchanan TS. Lower extremity kinematic and kinetic differences in runners with high and low arches. J Appl Biomech. 2001;17(2):153-163.

11. Hamill J, Van Emmerik REA, Heiderscheit BC, Li L. A Dynamical Systems Approach to Lower Extremity Running Injuries. Clin Biomech. 1999;14(5):287-308.

12. Rodrigues P, Chang R, TenBroek T, Van Emmerik R, Hamill J. Evaluating the coupling between foot pronation and tibial internal rotation continuously using vector coding. $J$ Appl Biomech. 2015;31(2):88-94.

13. Silvernail JF, Boyer K, Rohr E, Bruggemann G-P, Hamill J. Running Mechanics and Variability With Aging. Med Sci Sport Exerc. 2015;47(10):2175-2180.

14. Heiderscheit BC, Hamill J, Van Emmerik RE a. Variablitiy of Stride Characteristics and Joint Coordination Among Individuals With Unilateral Patellofemoral Pain. J Appl Biomech. 2002;18:110-121.

15. Seay JF, Van Emmerik RE a, Hamill J. Influence of low back pain status on pelvis-trunk coordination during walking and running. Spine (Phila Pa 1976). 2011;36(16):E1070E1079. doi:10.1097/BRS.0b013e3182015f7c

16. Hamill J, Haddad J, Milner C, Davis I. Intralimb Coordination in Female Runners wtih Tibial Stress Fracture. In: Proceedings of the XXth Congress of Teh International Society of Biomechanics. Cleveland, OH; 2005. 
17. Kurz MJ, Stergiou N. Does Footwear Affect Ankle Coordination Strategies? J Am Podiatr Med Assoc. 2004;94(1):53-58. doi:10.7547/87507315-94-1-53

18. Sinclair J. The influence of minimalist, maximalist and conventional footwear on impact shock attenuation during running. Mov Sport Sci - Sci Mot. 2017;(95):59-64.

19. Sinclair J, Richards J, Selfe J, Fau-Goodwin J, Shore H. The Influence of Minimalist and Maximalist Footwear on Patellofemoral Kinetics During Running. J Appl Biomech. 2016;32(4):359-364. doi:10.1123/jab.2015-0249

20. Sinclair J, Fau-Goodwin J, Richards J, Shore H. The Influence of Minimalist and Maximalist Footwear on the Kinetics and Kinematics of Running. Footwear Sci. 2016;8(1):33-39. doi:10.1515/humo-2016-0010

21. Agresta C, Kessler S, Southern E, Goulet GC, Zernicke R, Zendler JD. Immediate and short-term adaptations to maximalist and minimalist running shoes. Footwear Sci. 2018;4280:1-13. doi:10.1080/19424280.2018.1460624

22. Aminaka N, Arthur K, Porcari JP, Foster C, Cress M, Hahn C. No Immediate Effects of Highly Cushioned Shoes on Basic Running Biomechanics. Kinesiology. 2018;50(1):0-7. https://hrcak.srce.hr/ojs/index.php/kinesiology/article/viewFile/6340/3368.

23. Chang R, Van Emmerik R, Hamill J. Quantifying rearfoot-forefoot coordination in human walking. J Biomech. 2008;41(14):3101-3105. doi:10.1016/j.jbiomech.2008.07.024

24. Batschelet E. Circular Statistics in Biology. London: Academic Press; 1981.

25. Nordin M, Frankel V. Biomechanics of the Knee. In: Nordin M, Frankel V, eds. Basic Biomechanics of the Musculoskeletal System. 3rd ed. Philadelphia: Lippincott Williams and Wilkins; 2001:176-201.

26. Nigg BM, Baltich J, Hoerzer S, Enders H. Running shoes and running injuries: mythbusting and a proposal for two new paradigms: 'preferred movement path' and 'comfort filter.' Br J Sports Med. 2015;49(20):1290-1294. doi:10.1136/bjsports-2015095054

27. Nigg BM, Vienneau J, Smith AC, Trudeau MB, Mohr M, Nigg SR. The Preferred Movement Path Paradigm: Influence of Running Shoes on Joint Movement. Med Sci Sports Exerc. 2017;(15):1641-1648. doi:10.1249/MSS.0000000000001260

28. Boyer KA, Freedman Silvernail J, Hamill J. Age and sex influences on running mechanics and coordination variability. J Sports Sci. 2017;35(22):2225-2231.

doi:10.1080/02640414.2016.1265139

29. Bonacci J, Saunders PU, Hicks A, Rantalainen T, Vicenzino B (Guglielmo) T (Guglielmo) T, Spratford W. Running in a minimalist and lightweight shoe is not the same as running barefoot: A biomechanical study. Br J Sports Med. 2013;47(6):387-392. doi:10.1136/bjsports-2012-091837

30. Sinclair J, Atkins S, Taylor PJ. The Effects of Barefoot and Shod Running on Limb and Joint Stiffness Characteristics in Recreational Runners. J Mot Behav. 2016;48(1):79-85.

31. Kong PW, Candelaria NG, Smith DR. Running in new and worn shoes: A comparison of three types of cushioning footwear. Br J Sports Med. 2009;43(10):745-749. 
doi:10.1136/bjsm.2008.047761

32. Wang L, Li JX, Hong Y, Zhou JH. Changes in heel cushioning characteristics of running shoes with running mileage. Footwear Sci. 2010;2(3):141-147.

doi:10.1080/19424280.2010.519348

33. Floría P, Sánchez-Sixto A, Ferber R, Harrison AJ. Effects of running experience on coordination and its variability in runners. J Sports Sci. 2018;36(3):272-278. doi:10.1080/02640414.2017.1300314

34. Hafer JF, Peacock J, Zernicke RF, Agresta CE. Segment Coordination Variability Differs by Years of Running Experience. Med Sci Sports Exerc. 2019;51(7):1438-1443. doi:10.1249/MSS.0000000000001913

35. Nakayama Y, Kudo K, Ohtsuki T. Variability and fluctuation in running gait cycle of trained runners and non-runners. Gait Posture. 2010;31(3):331-335.

doi:10.1016/j.gaitpost.2009.12.003 
Table 1. Coupling Angle Bin Definitions

\begin{tabular}{lc}
\hline Coordination Pattern & Coupling Angle Bin Definition \\
\hline Anti-phase (i) & $112.5^{\circ} \leq r<157.5^{\circ}, \quad 292.5^{\circ} \leq r<337.5^{\circ}$ \\
In-phase (ii) & $22.5^{\circ} \leq r<67.5^{\circ}, \quad 202.5^{\circ} \leq r<247.5^{\circ}$ \\
Proximal phase (iii) & $0^{\circ} \leq r<22.5^{\circ}, \quad 157.5^{\circ} \leq r<202.5^{\circ}, \quad 337.5^{\circ} \leq r<360^{\circ}$ \\
Distal phase (iv) & $67.5^{\circ} \leq r<112.5^{\circ}, \quad 247.5^{\circ} \leq r<292.5^{\circ}$ \\
\hline
\end{tabular}


Table 2. Mean Values ( \pm standard deviation) of Rearfoot, Tibia, and Knee Kinematics During the Stance Phase for Minimalist Shoe Conditions, Neutral Shoe Conditions, and UltraCushioning Shoe Conditions

\begin{tabular}{lccccc}
\hline & Time & Minimalist & Neutral & Ultra & $p$ \\
\hline \multirow{4}{*}{ Rearfoot } & IC & $0.88 \pm 4.00$ & $1.94 \pm 4.54$ & $0.67 \pm 5.42$ & .604 \\
& Peak & $-10.00 \pm 3.45$ & $-9.29 \pm 4.13$ & $-9.11 \pm 5.74$ & .785 \\
& ROM & $10.88 \pm 2.49$ & $11.22 \pm 2.83$ & $9.78 \pm 3.01$ & .392 \\
\multirow{4}{*}{ Tibia } & IC & $5.38 \pm 4.27$ & $4.0 \pm 7.03$ & $2.09 \pm 8.32$ & .286 \\
& Peak & $0.638 \pm 4.64$ & $-2.40 \pm 6.00$ & $-4.29 \pm 8.34$ & .082 \\
& ROM & $4.74 \pm 4.12$ & $6.40 \pm 2.81$ & $6.38 \pm 2.65$ & .399 \\
\multirow{4}{*}{ Knee } & IC & $-13.92 \pm 5.22$ & $-13.30 \pm 5.71$ & $-10.73 \pm 5.15$ & .099 \\
& Peak & $-36.15 \pm 5.41$ & $-36.04 \pm 6.47$ & $-33.55 \pm 5.57$ & .066 \\
& ROM & $22.23 \pm 4.01$ & $22.74 \pm 3.74$ & $22.82 \pm 3.22$ & .784 \\
\hline
\end{tabular}


Figure 1.

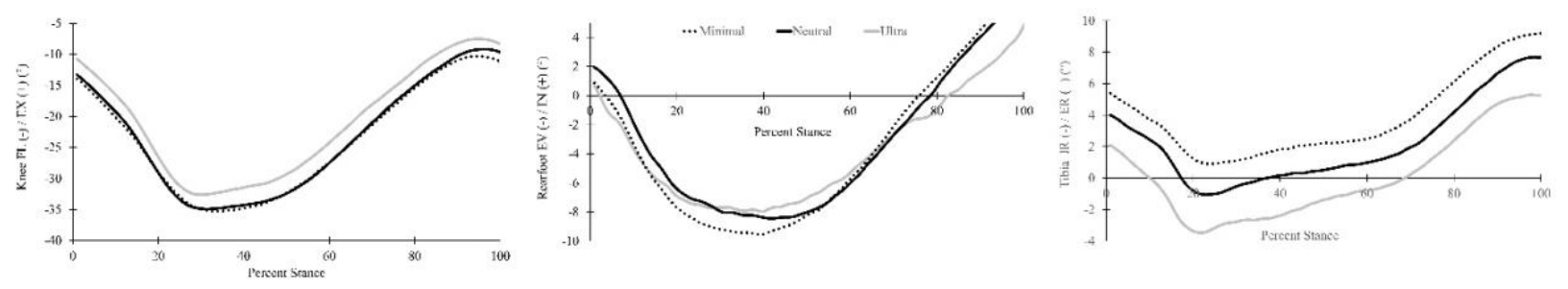


Figure 2
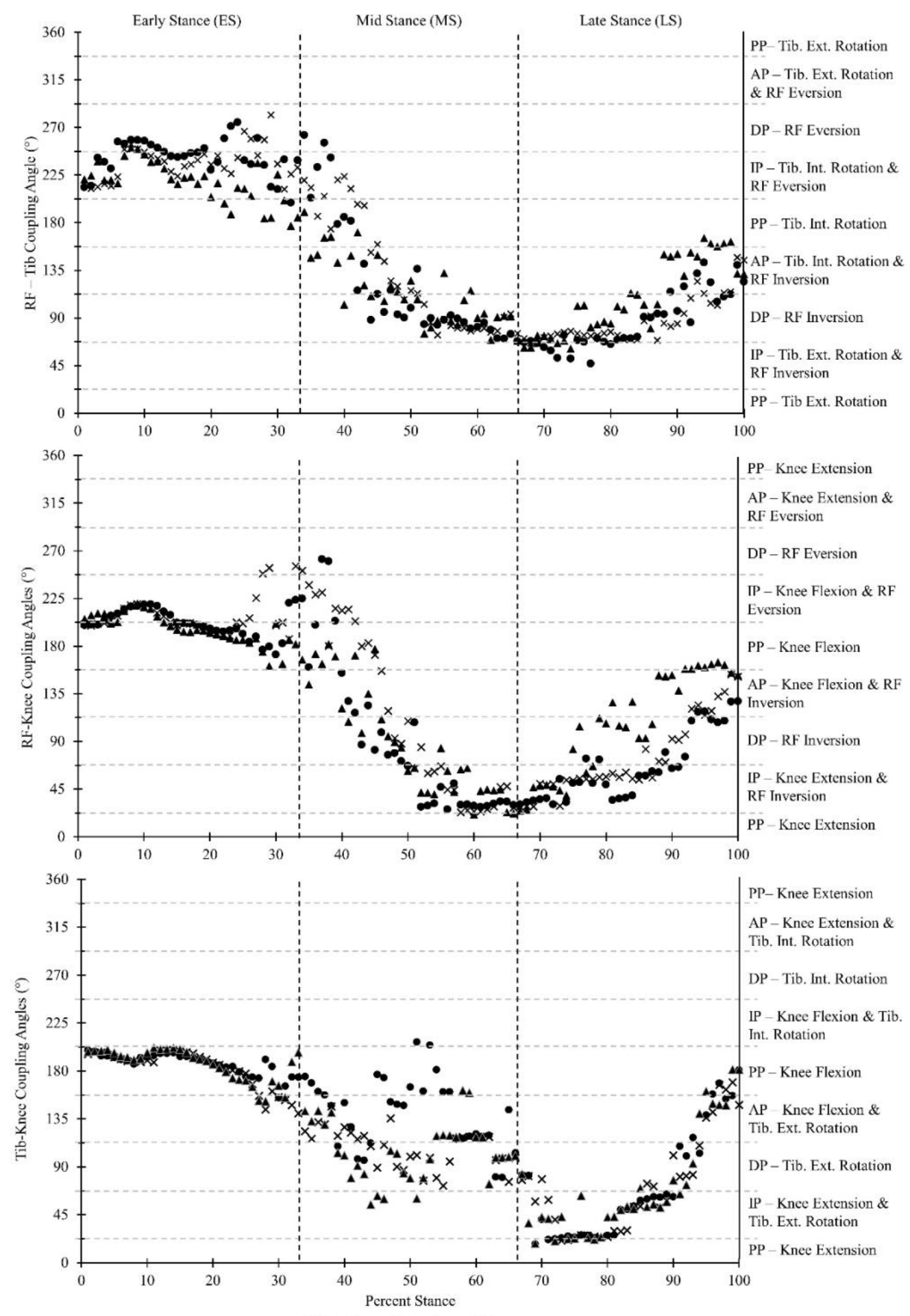
Figure 3

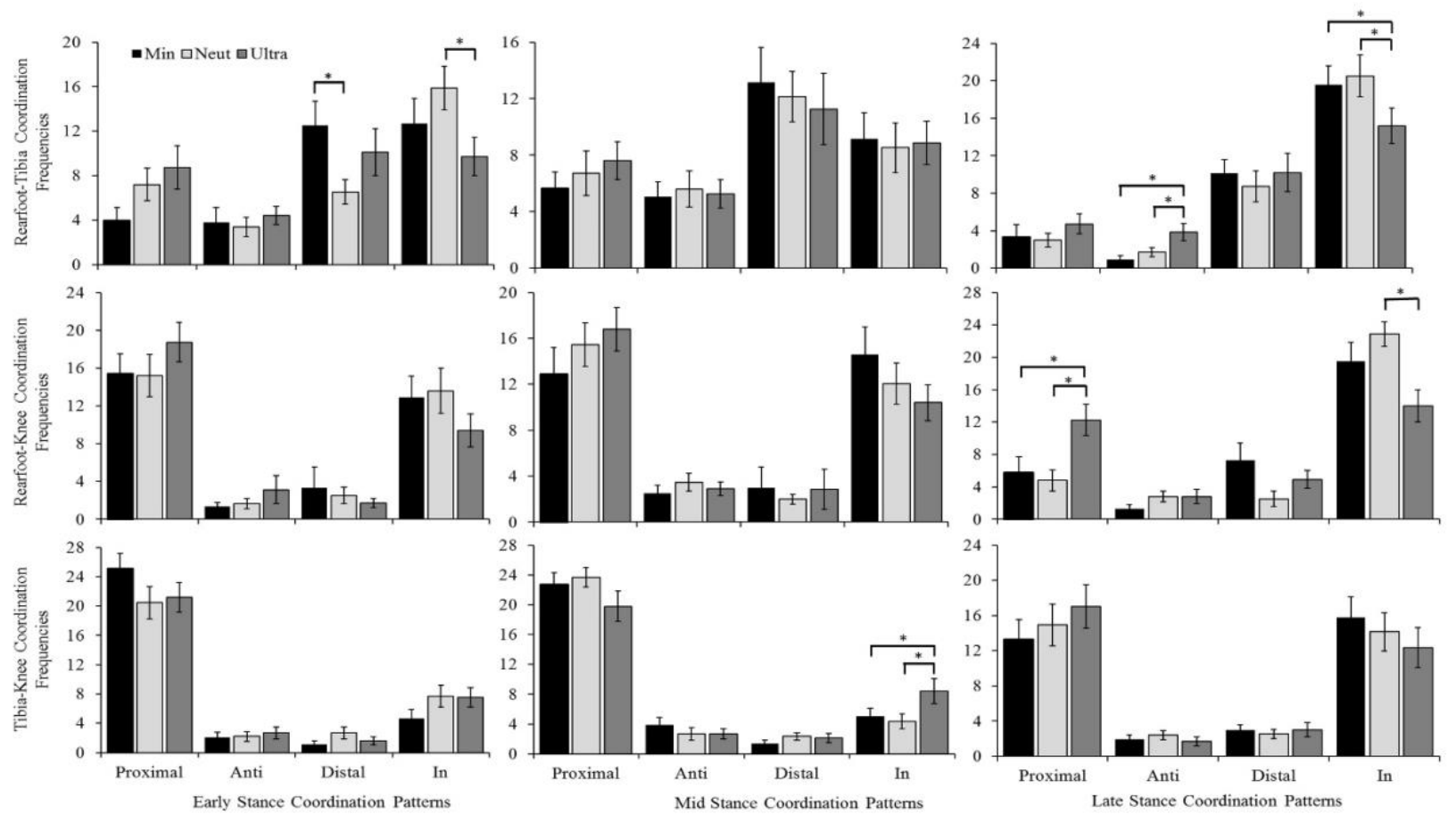


Figure 4
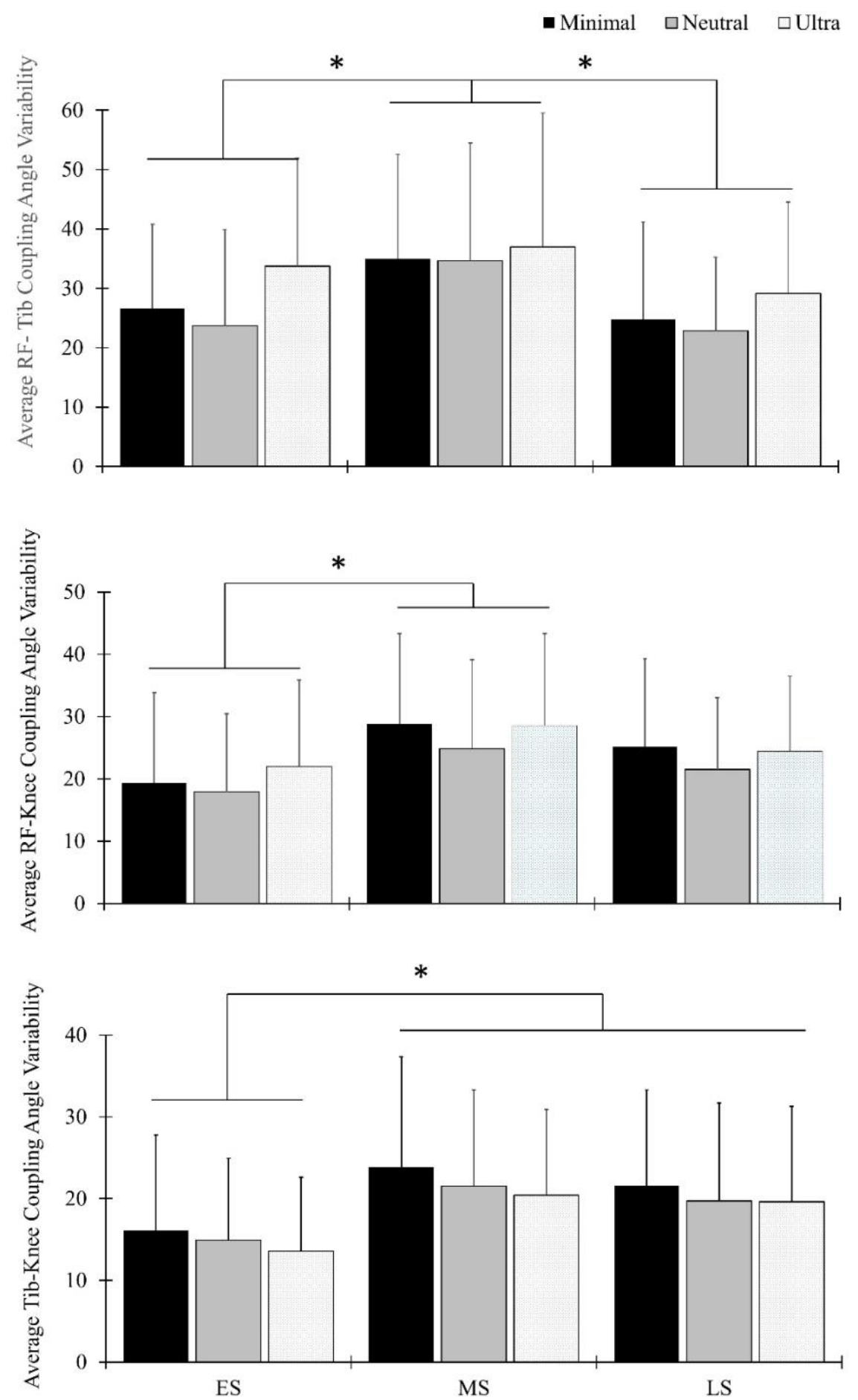


\section{Figure Captions}

Figure 1. Mean angles across the stance phase for knee flexion/extension (left graph), rearfoot eversion/inversion (middle graph), and tibia internal/external rotation (right graph).

Figure 2. Coupling angles across stance for rearfoot (RF)-tibia (Tib) (top), rearfoot-knee (middle), and tibia-knee (bottom). Coordination patterns along with their respective kinematics are shown on the right of each graph. PP indicates proximal phase, AP anti-phase, DP distal phase, IP in-phase, RF indicated rearfoot, Tib. indicates tibia. The kinematic motions describe what joint motion dominated the coordination pattern, i.e. distal phase with the dominant motion being rearfoot eversion, proximal phase with the dominant motion being knee flexion, or inphase with equal amounts of rearfoot eversion and knee flexion..

Figure 3. Frequency counts for rearfoot-tibia (top row), rearfoot-knee (middle row) and tibiaknee (bottom row) grouped by early stance (ES, left column), middle stance (MS, middle column), and late stance (LS, right column). * indicates significant difference at $p<0.05$ level.

Figure 4. Average coupling angle variability for rearfoot-tibia (top), rearfoot-knee (middle), and tibia-knee (bottom). * indicates significant difference at $p<0.05$ level. 